BULLETIN Bulletin hispanique

HISPANIQUE Université Michel de Montaigne Bordeaux

$111-2 \mid 2009$

Varia

\title{
Realidades imbricadas y seres desdoblados
}

La vida como mise-en-abyme en Jindama, de Alfonso Vallejo

John P. Gabriele

\section{OpenEdition}

Journals

Edición electrónica

URL: http://journals.openedition.org/bulletinhispanique/1048

DOI: 10.4000/bulletinhispanique. 1048

ISSN: 1775-3821

Editor

Presses universitaires de Bordeaux

Edición impresa

Fecha de publicación: 1 diciembre 2009

Paginación: 639-653

ISBN: 978-2-86781-617-8

ISSN: 0007-4640

Referencia electrónica

John P. Gabriele, « Realidades imbricadas y seres desdoblados », Bulletin hispanique [En línea],

111-2 | 2009, Publicado el 01 diciembre 2012, consultado el 20 abril 2019. URL : http://

journals.openedition.org/bulletinhispanique/1048 ; DOI : 10.4000/bulletinhispanique.1048 


\title{
Realidades imbricadas y seres desdoblados: la vida como mise-en-abyme en Jindama, de Alfonso Vallejo
}

\author{
John P. Gabriele \\ The College of Wooster - Ohio - USA
}

Jindama (1998) présente un univers de situations et de personnages sur divers plans artistiques de manière à mettre en cause la connaissance ontologique et à révéler que l'art et la vie constituent des processus de re-textualisation et de réappropriation qui s'autoréfléchissent et s'auto-engendrent.

Jindama (1998) representa un mundo de situaciones y personajes de distintos planos artísticos con el fin de poner en tela de juicio el conocimiento ontológico y revelar que el arte y la vida son procesos de retextualización y reapropriación auto-reflexivos y autogenerativos.

'Jindama' (1998) depicts a world of situations and characters on distinct artistic planes, in order to call into question ontological knowledge and reveal that art and life are processes of self-reflective and auto-generative re-textualization and re-appropriation.

Mots-clés: Mise-en-abyme - Identité - Posmodernité - Auto-réflexifs - Autogénératifs.

$\mathrm{E}$ L TEATro de Alfonso Vallejo (1943) es distintivamente antirrealista e incisivamente experimental (ver Martín 152, Coco 221-24, Gutiérrez Carballo 18-19). El dramaturgo desafía persistentemente los límites de

$B H i$, Tome 111, nº 2 - décembre 2009 - p. 639 à 653. 
la representación artística en sus obras con intenciones decididamente ontológicas. El universo teatral vallejiano gira alrededor de un eje teórico y formal principal: la certeza que lo ilusorio y lo real son conceptos igualmente autoritarios y tenues. La realidad y la ilusión se imponen con igual autoridad y fuerza en las obras del dramaturgo para dar la impresión de que el arte teatral y la vida son procesos de retextualización y reapropriación esencialmente auto-reflexivos y auto-generativos. El mundo teatral de Vallejo no ofrece paralelos explícitos ni implícitos con el mundo exterior del espectador. La inestabilidad y fugacidad que caracterizan sus textos tienen como objetivo último asentar la autonomía del proceso artístico creativo y hacer frente a la supuesta preeminencia empírica de la realidad. Como resultado, sus personajes -y también sus espectadores- terminan descentrados, incapaces de arraigarse frente a sus respectivas circunstancias.

Jindama (1998) es un texto teatral vertiginosamente laberíntico en el cual se confluyen y (con)funden situaciones y personajes de distintos planos artísticos para crear una obra agudamente paradójica que se deshace y se integra simultáneamente mientras evoluciona la acción. Leer la obra o verla montada constituye un ejercicio estético e intelectual desafiador. La forma y el contenido del drama desafían a los lectores/espectadores. Vallejo nos obliga a interaccionar con su texto y a ejercer nuestras habilidades detectivescas para conectar productivamente los diversos elementos de su obra.

La pieza es la fusión de tres textos distintos. Cada texto es protagonizado por personajes distintos que no trascienden nunca los confines del escenario propio. Los textos se imbrican y los personajes se desdoblan creando un mundo híbrido en que se transita consecuentemente entre pasado, presente y futuro, historia y fantasía, vida y arte. Jindama, como vamos a ver, es la dramatización de los conceptos principales del arte posmoderno: «anarchy, anti-narrative, indeterminacy, deconstruction of a world-model, and ontological uncertainty» (Lethen 235).

La contextura posmoderna de Jindama se establece mucho antes de empezar la acción mediante lo que anota Vallejo sobre el esquema dramático, los personajes, la escenografía y la acción. «La acción», escribe el dramaturgo (13), «discurre en tres niveles. Por un lado, el actoral. Cuatro actores, dos hombres (Klaus y Marco) y dos mujeres (Reme y Gina) llegan a una escena para ensayar 'Jindama' bajo la dirección de Lev». "Por otro lado», sigue Vallejo, «estos actores interpretan personajes». Klaus, Marco, Reme y Gina desempeñan los papeles de Juanaco, Troncho, Reme y Tora en la pieza que van a ensayar. "Juanaco» además, "se ha convertido en un lector apasionado de Cicerón». La vida del escritor romano "le ha impactado de tal forma» que «le ha hecho confundir realidad y ficción» y a veces se cree Cicerón. 
Por lo tanto, en el mundo fantaseado de Juanaco, Troncho, Reme y Gina se convierten en Marco Antonio, Sempronia y Cleopatra (13).

Mientras evoluciona la acción, los tres planos se entrecruzan a nivel contextual con el fin de poner en tela de juicio el conocimiento ontológico. En cuanto al drama propio de Vallejo, ha de notarse que los personajes se identifican exclusivamente «con el nombre del personaje que van a interpretar en la supuesta obra que se está montando» (17). Pero cuando hablan entre ellos, utilizan sus nombres de actores lo cual sugiere que la realidad exterior, la que existe más allá de los confines del escenario, o sea la realidad de los actores y espectadores de la obra de Vallejo y, por cierto, del mismo Vallejo, no es la fuente del acto teatral mimético de su obra. La realidad en Jindama, como diría Jean Baudrillard, "has effectively vanished and all that is left is simulations" (170). Desde el principio se nos da a entender que Jindama se auto-genera y se difunde sin ningún apoyo ajeno, que las estructuras anquilosadas del realismo cotidiano y dogmático ya no sirven, hecho que se adelanta en la descripción del escenario y de la acción. "La escena», escribe Vallejo, «representa la escena vacía de un teatro. ... Las paredes de la supuesta escena donde se va a desarrollar la acción ... se pueden indicar únicamente por efectos de luz y de sonido. Unidad de lugar» (18). «La acción transcurre en cuadros», especifica el dramaturgo, "de forma continuada, sin interrupciones» (19).

Igual que los múltiples papeles de los personajes, la descripción del escenario y de la acción son indicios de la desaparición de la línea divisoria entre lo real y lo ilusorio asociada con la creación artística convencional. El espacio y el tiempo han perdido su capacidad de delimitar y concretar la acción para asegurar, como diría Linda Hutcheon que en Jindama no hay "any sure ground upon which to base representation and narration" (92). Después de apuntar que "la acción discurre en tres niveles», luego sugerir que sus personajes son seres poliédricos y por fin insistir en que la acción se sitúa en un solo lugar, en que ese lugar es la representación de «una escena vacía», y en que la acción transcurre sin interrupciones, Vallejo deja tajantemente claro que los personajes de su drama son el producto de una coalescencia enredosa de niveles convergentes de arte y un continuo artístico espaciotemporal sin índices claros de referencia.

En Jindama, Vallejo nos invita a contemplar una construcción del sujeto teatral fundamentalmente alternativa. Si el personaje teatral tradicional es el resultado de la integración de fuerzas históricas, sociales y políticas, en Jindama es el agregado de impulsos puramente artísticos. Los actores/ personajes de Vallejo son seres fracturados en el mejor sentido de la palabra. No son actores que representan ilusiones teatrales, sino actores que las viven. 
Ejemplifican lo que dice June Schlueter de los personajes metateatrales: «the rift between the essential self (the actor) and the role-playing self (the character)" es sintomático de "the loss of identity» (14). Klaus "encarna a Juanaco, antiguo cantaor flamenco fracasado, que posteriormente se ha convertido en asesor fiscal» (13) y que se cree «a veces Cicerón» (14). Marco, quien juega el papel de Troncho en la obra que están ensayando, es también Marco Antonio en el antiguo mundo romano fantaseado de Juanaco. Reme se conoce por el mismo nombre como actriz en la Jindama de Vallejo y como personaje en la Jindama que los actores han venido a ensayar. También es Sempronia, la mujer de Cicerón. Gina, la actriz que desempeńa el papel de Tora en el drama que están ensayando, es también Cleopatra en la realidad delirante de Juanaco. Sólo Lev desempeña un papel, el de director. Los personajes de Jindama y sus acciones reflejan una deliberada preferencia de "performance over representation», como diría Jeanette Malkin, para subrayar la inestabilidad del «yo» (148). Mediante la construcción de sus personajes en Jindama, Vallejo logra oponerse a la noción convencional del ser teatral auténtico y unificado.

Los nombres que llevan los personajes también subrayan el estado de flujo en que existen. Tradicionalmente el nombre sirve para concretar la existencia y la identidad del individuo según Thomas Docherty. En palabras de Docherty, "to say a name is to totalize an existence» (50). El acto de nombrar tiene tres funciones. Primero, "the name indicates authority... a kind of ontological authority». Segundo, el nombre sirve como "a locus around which characterization actually takes place» $\mathrm{y}$, tercero, le ofrece al lector «a point of view on the fiction as a whole, ... a position for the reader to inhabit and from which to see the world of the fiction» (73-74). Sucintamente dicho, el nombre otorga estabilidad y constancia textual, contextual y formal. Pero los nombres de los personajes de Jindama tienen todo lo contrario como objetivo. Reflejan una perspectiva decididamente pluralista frente al personaje.

Klaus y Gina se conocen por tres nombres distintos en los tres textos. Reme, sin embargo, lleva el mismo nombre en dos dramas del mismo título, la Jindama de Vallejo y la obra interior que dirige Lev, y tiene un nombre distinto en el mundo delirante de Juanaco. Comparablemente, Marco tiene el mismo nombre en la pieza propia de Vallejo y en la realidad imaginada de Juanaco, pero no en el drama que están ensayando. Aunque Lev, el director y único personaje que se conoce por un solo nombre, no parece reflejar la esquizofrenia fundamental encarnada en los otros personajes, sí da muestras de ser un personaje inestable e indefinido: «Se maquilla la cara, pero sin componer un personaje concreto" (17). Los nombres de los personajes y 
los varios papeles que desempeñan son señales de una existencia prismática y de una desintegración formal. El fin de Vallejo es contrarrestar cualquier posibilidad de construir una totalidad significante, producir una visión unificadora y negarles a sus lectores/ espectadores el puesto tradicional de contemplación estable y cómodo frente a la obra de arte.

Jindama es una obra de mundos contiguos y confluentes que pone en duda la autoridad narrativa mediante la representación de la condición humana como una mise-en-abyme, como "a series of apparently endlessly overlapping, enclosed networks of conceptual or structural space which form a kind of labyrinth leading to a shifting, ever-unattainable nucleus or centre» (Cardwell 271). La existencia abismal de los personajes se refleja inicialmente en las relaciones que llevan. En la obra que se está ensayando, Juanaco y Reme son marido y mujer. Reme, actriz, es la pareja de Marco, actor. Pero en el papel de Sempronia, es la mujer de Cicerón. Troncho y Tora son novios en la pieza que ensayan, relación que hace paralelo con la que llevan como Marco Antonio y Cleopatra en el mundo delirante de Juanaco. Lev, el director lleva una relación con Gina. Vivir en una mise-en-abyme es existir en un mundo carente de una realidad autoritaria, absoluta y orientadora. Es vivir en ilusiones que sólo suponen ser realidades, en más de una realidad y en ninguna al mismo tiempo. Es vivir en varios mundos que se convergen y divergen y que ocasionan el fraccionamiento y la inmolación eventual del ser como en el caso de los personajes de Jindama.

$\mathrm{Si}$ he analizado minuciosamente lo que proporciona Vallejo sobre sus personajes y la escenografía y la acción en su esquema dramático, lo he hecho porque aporta claves teóricas indispensables para el análisis de Jindama, como observa Francisco Gutiérrez Carbajo (111), y también para insistir en la inherente posmodernidad de la pieza. Como bien se sabe, todo artista posmoderno concibe sus obras como tratados teóricos y exposiciones textuales (ver Malpas 1-9 y Schulte-Sasse). Típicamente, los autores posmodernos formulan postulados teóricos que reafirman mediante la práctica en sus textos, aserción adicional de la auto-reflexión y auto-generatividad de su arte. Jindama, como los dramas posmodernos en general, es un texto esencialmente auto-suficiente y autónomo.

Jindama no se conforma a ningún diseño impuesto desde fuera. Textual y contextualmente, es un drama que se sostiene independientemente como reafirma el intercambio entre Juanaco y Troncho con que se da inicio a la acción del primer cuadro. "iJindama! Sí. .. me da jindama. No me gusta. Me da miedo», dice Juanaco. Troncho contesta: «El primer ensayo con decorado siempre desconcierta» y reincide en el aspecto amenazante de la puerta: 
«Parece que te va a morder» (21). Luego pregunta sobre el director y el autor de la obra que van a ensayar, a los cuales Juanaco llama «gilipollas» (22).

El primer intercambio entre Juanaco y Troncho reitera que la acción de Jindama se contextualiza por la falta de causalidad y consistencia, pone en cuestión la autoridad de la dirección y la autoría teatral y distancia al propio Vallejo de su obra. La obra a que se refieren Juanaco y Troncho no es la obra que han venido a ensayar de acuerdo con lo que se aprende en el esquema dramático, sino el texto fantaseado de Juanaco, señal que el texto de Vallejo se rige por una falta de lógica discursiva sobre la cual el propio dramaturgo no tiene control. Al ser "Jindama» la primera palabra que se pronuncia implica que los personajes son conscientes de ser creaciones artísticas y que, metafóricamente hablando, dar título a la obra dramatizará sus vidas. Por lo visto, la autoridad narrativa de Jindama se origina dentro del texto; no se impone desde fuera. Esto en combinación con la repudiación categórica del autor y del director teatral, "gilipollas» según Juanaco, sugiere que no hay más allá del escenario un mundo real, sino únicamente un teatro que se contempla a sí mismo.

El drama de Vallejo, la pieza que va a dirigir Lev y el mundo fantaseado de Juanaco están indistinguiblemente fusionados. Los personajes existen en la incómoda encrucijada de planos ilusorios que se entrecruzan naturalmente como se confirma a continuación. Mientras hablan Troncho y Juanaco, éste pregunta: «¿Qué te gustaría?» Troncho responde: "Cortarte la cabeza». Juanaco reacciona: « $i$ Chico, te has metido en el personaje de una forma!» (25). La respuesta de Troncho corresponde contextualmente al antiguo mundo romano fantaseado de Juanaco, no al drama de Vallejo ni a la pieza interior, lo cual supone la superposición de varias ilusiones igualmente autoritarias como advierte el mismo Troncho. Le pregunta a Juanaco: «№ te parece suficiente contradicción que tú, que te llamas Klaus, hagas un personaje que se llama Juanaco, cantaor frustrado de Jerez, metido a asesor fiscal y que se vuelve loco y se cree Cicerón?» Juanaco contesta: "Teatro dentro del teatro del teatro. Metateatro o postmetateatro, según se mire» (26). La escena hace visible lo que logra comunicar Vallejo mediante su meticulosa descripción de los personajes, que la vida es "the product of an arbitrary discursive organization» de textos y contextos (Murphy 260) y que es imposible saber dónde un texto o contexto «ends and another begins» (Waugh 29).

Haciendo hincapié en la imposibilidad de separar las ficciones que constituyen la obra de Vallejo, Troncho exclama: «iPero te lo quiero advertir, Klaus! ¡En esta obra... a la menor que me hagas... a la menor... cuando llegue la escena de la decapitación... la pagas!» (29). El acto violento a que se refiere Troncho es la decapitación de Cicerón por Marco Antonio 
en el mundo maquinado de Juanaco. Sin embargo, las consecuencias del acto corresponden a su realidad como actores. «Dime una cosa Klaus», dice Troncho, "¡a que no sabes por qué he aceptado el papel de Marco Antonio? ... Porque te tengo que cortar la cabeza. ... Y yo te tengo que cortar la cabeza» (33-34).

Apoderado por la actuación, Troncho se pierde en la ilusión. «Exaltado, en romano asesino», grita: «Y Ylegan los sicarios de Marco Antonio a su villa de Formio, el tribuno Antonio y el centurión Herenio... !» (36). Hace gesto de poner el cuello, como Cicerón, "para que le corten la cabeza», y se oye un "golpe terrible imitando la decapitación de Cicerón». Despavorido, Juanaco reacciona: «¡Pones cara de asesino! ¡De sádico! ¡Parece que estás imaginando mis carótidas sangrando, mi cabeza sujetada por un hilo de carne a la columna vertebral». Troncho responde con tono irónico: "¿De qué quieres que ponga la cara, querido? ¡De Marco Antonio! ¡Estoy actuando!» (37). Juanaco no tarda en insistir que hay que cambiar la escena de la decapitación aunque Troncho le asegura que «¡ . . todo es mentira! ¡Pura ficción!» (43). Pero en un mundo carente de realidad concreta y estable, la pura ficción se convierte en realidad.

El primer cuadro de Jindama dramatiza la lucha inútil por separar el arte y la vida. Como todo personaje metateatral, Juanaco y Troncho revelan cierta conciencia de su profesión y sus existencias teatrales. Según, N. M. Rao, "the characters of metadrama not only comment on techniques but on themselves» (220). El intercambio entre Juanaco y Troncho infiere que la ilusión o la ficción y el arte o la realidad constituyen asuntos técnicos y metafísicos en Jindama. Consistente con la imagen de la puerta amenazante que impide cualquier salida del escenario, Juanaco y Troncho son prisioneros del arte en el más estricto sentido de la palabra. Dada su existencia dual como actores y personajes, desempeñan sus papeles y comentan sobre ellos al mismo tiempo. Participan en su propia dramatización y acaban teatralizando sus vidas como si fuera la cosa más natural.

Lev entra por primera vez en el Cuadro II. Su interacción con Juanaco proporciona otra oportunidad para subrayar la existencia abismal de los personajes de Jindama. Cuando Juanaco le informa a Lev que Troncho «¡Me quiere decapitar!», Lev pregunta: «¡Y qué hay de malo en eso?» (45). Consciente del dilema existencial con que se ve enfrentado, Juanaco le explica: «[Y]o, Klaus, tiene que interpretar a Juanaco, que ha sido un antiguo cantaor de flamenco y que se ha metido a asesor fiscal, que se ha vuelto loco leyendo a Cicerón y se cree que es Cicerón... ». Lev responde en términos propios del metateatro y de la posmodernidad: «[A]hora ... precisamente ahora estás entrando en tu papel, Klaus. ... ¡Ese es el camino, Klaus! ¡La ruta 
de la ambivalencia del ser, de la ambigüedad existencial, de la cornisa, del precipicio existencial, del ser o no ser... i" (49-51).

La inestabilidad del personaje de Juanaco se refleja en el escenario movedizo: "[L]as paredes se mueven, se iluminan, se transparentan, como movidas por un aire que las hincha y deshincha. ... El espacio de la escena se vuelve de pronto octogonal, después poliédrico, se achica, se agranda» (51). El intercambio entre Juanaco y Lev y la descripción del escenario siguen recalcando la auto-generatividad del drama de Vallejo y la falta total y efectiva de una conexión extratextual.

Lo que transcurre en Jindama denota una ruptura con la concepción unidimensional y fijeza perspectivista del arte teatral convencional. En Jindama, "todo está unido», como acierta Juanaco. "Forma un bloque existencial» (61). Las palabras de Juanaco hacen eco a lo que dice Nicholas Kaye de los textos posmodernos, que son "a form of "bricolage" in which narrative development is displaced and undermined» (12), que son textos en que se contraponen "narrative elements ... in such a way that the event of narration, the move toward containment, is frustrated" (139). La estructura de Jindama conlleva consecuencias existenciales para los personajes. Existir en el entrecruce de textos y contextos termina por derrumbar la jerarquía estética y filosófica tradicional entre actor y personaje teatral y disuelve la distinción entre el «yo» y la «otredad». Al crear personajes que son incapaces de saber con certidumbre en cuál de los tres planos artísticos actúan, Vallejo logra demostrar que la identidad es «a performance that is indefinitely mutable rather than being based on some essential nature» (Malpas 74). Los personajes de Jindama, igual que el drama de Vallejo, carecen de un núcleo esencial y estabilizador, de un eje fijo y consolidado.

Existir en un mundo carente de fronteras es existir en un estado de infinita regresión y repetición; es estar atrapado en una puerta giratoria que va en direcciones opuestas simultáneamente, hecho de que Troncho es consciente: "[N]o sé... no sé cómo vamos a salir de aquí» (53). Se trata de un mundo donde el ser se desmenuza incontrolablemente, donde se transforma perpetuamente, se quiera o no se quiera: "Aparece de pronto Lev, ya en vías de transformación, con la mitad de la cara pintada, con ojos terribles, la otra mitad de la cara, con rasgos cómicos» (54). El mundo de Jindama es un mundo hermético e impenetrable desde fuera, un mundo gobernado por las intrigas de lo ilusorio que anula toda posibilidad de existir fuera de los confines del escenario: «De pronto Klaus amaga, la puerta se abre, Klaus quiere salir de la escena, intentando coger a la puerta a traición, pero la puerta se cierra de golpe» (64). Una vez encerrado en el mundo de la ilusión, no hay posibilidad de volver al exterior: «Por un lateral aparece Reme. ... 
Bruscamente la puerta se abre de golpe y Reme queda frente a Klaus y Marco. ... La puerta se cierra bruscamente» (65-66).

La identidad en Jindama está fraccionada. Los personajes se mueven por un mundo laberíntico, inestable y movedizo donde son múltiples seres y ninguno al mismo tiempo como reafirma Reme: «[N]o estoy en el personaje ... i ... soy Tora, la esposa de Juanaco y al mismo tiempo Sempronia, la esposa de Cicerón!» (71). El personaje en Jindama, como diría Stuart Sim, "has no essential core of identity, and is to be regarded as a process in a continual state of dissolution rather than a fixed identity" (367). Es el producto de contextos idénticos y distintos como infiere lo que le dice Troncho a Juanaco: «Lo que más me fastidia en ti es que miras a mi mujer como si no fuera mi mujer sino nuestra mujer...» (76). Todos son conscientes de su fraccionamiento, de su existencia fundamentalmente teatral. "¿Por qué tanta representación de representación... ?», pregunta Reme. «Yo, Reme, y la mujer de Juanaco y Sempronia, la mujer de Cicerón... ¿por qué tantos planos?» (84). Lev, el portavoz del propio Vallejo, contesta y reafirma la teoría posmoderna que impulsa lo que transcurre en Jindama: «Porque la realidad es una elaboración móvil, múltiple y cambiante. ... porque es una metáfora de la interpretación humana...» (84-85). El intercambio entre Reme, Lev y Troncho confirma que Vallejo, como seguramente diría Robert Corrigan, utiliza «a theatrical context to create a lifés experience» (156). Cuando Lev explica que «se trata de una obra abierta» (85), Troncho subraya una vez más la estructura prismática del drama y de sus vidas: “¡No es que esté abierta! Es que está tan abierta que se va por todas partes...» (86).

Si representar más de un papel es la base de la existencia en Jindama, el acto de ensayar es indispensable para sobrevivir como declara Lev: "Voy al camerino a prepararme. Que yo también actúo... Y tengo que ensayar» (53). En un mundo donde no se distingue entre vida y arte, entre actor y papel, entre actuar y vivir, ensayar tiene una función artística y una función vital lo cual explica el deseo ansioso de los personajes por ensayar. "Pues a ver cuándo empezamos a ensayar», insiste Troncho (72). "Queremos saber qué estamos representando", declara Juanaco (74). Todos quieren averiguar su razón de ser en términos teatrales, como indican las palabras de Reme: «iMe quiere decir alguien cuándo empezamos a ensayari ... Necesitamos saber ... qué hacemos aquí» (82-83). Envueltos en un juego aleatorio de planos contiguos y contingentes, los personajes de Jindama anhelan establecer su "yo», aspiran a desempeñar el papel de su verdadero ser.

El ensayo de Jindama, que por fin comienza en el Cuadro IV, marca la culminación del dilema ontológico de los personajes $\mathrm{y}$ "the problem of the relation between the game-like artifices of fiction and the problematic imitation 
of reality» (McHale 62). El Cuadro IV confirma que el drama propio de Vallejo, la pieza que dirige Lev y la realidad fantaseada de Juanaco forman un tríptico cuyos tres cuadros se han doblado uno sobre el otro creando una fusión contextual que da origen a personajes que existen en tres planos artísticos simultáneamente. Cuando Klaus, quien representa el papel de Juanaco, insiste en que "Cicerón soy yo» (92), Tora reacciona en términos propios de la condición posmoderna: «Te crees Cicerón, pero en el fondo no te lo crees. Y tampoco te crees Juanaco ... ni tampoco Klaus ... Te crees ... uno y muchos al mismo tiempo ... o quizás ninguno en concreto ...» (93). Todos parecen ser conscientes de su disolución. Cuando Juanaco se dirige a Tora llamándola Cleopatra, ésta pregunta «¡Tora o Cleopatra?» y Juanaco responde, «Gina, Tora o Cleopatra... como quieras... ya ... da igual... Aquí hay más planos de la realidad que en la Escuela de Arquitectura» (98).

La fundición de Klaus-Juanaco-Cicerón y Gina-Tora-Cleopatra es el resultado de la (con)fusión de los tres planos artísticos distintos. Los personajes de Jindama tipifican los personajes pluralistas de obras posmodernas cuya caracterización mina «the principles of characterological causality and consistency" (Bertens 148-49). Ya no se trata de personajes que juegan múltiples papeles, sino de personajes que existen sin diferenciar entre sus distintos seres. Cuando Troncho, confundido, le pregunta a Juanaco: «¡Tú eres Juanaco, el jefe de Estafasa?», Juanaco contesta: « Ese soy!» Cuando Troncho vuelve a preguntar: "¿Y también Cicerón?», Juanaco responde»: ¡Correcto!» (113). Los personajes de Vallejo, como los textos y contextos que se entretejen en su drama, se doblan, se triplican, se repiten, y se reflejan indeterminadamente hasta aniquilar sus «yoes» auténticos como declara el mismo Juanaco: «Me están despersonalizando» (99).

Mientras evoluciona la acción, Vallejo sigue recalcando la muerte del autor y del texto escrito como reflejo de la auto-generatividad de su texto y con fines decididamente ontológicos. Cuando Lev anuncia que el ensayo de Jindama ha terminado, "ninguno de los actores obedece al director». Los actores, descontrolados, siguen interpretando por su cuenta. Lev grita «¡Texto!» e insiste que "¡No podemos irnos del texto!» Pero los actores no le hacen caso. "Hay que seguir... seguir...», dice Juanaco» (108). Más insiste Lev en imponer la autoridad del texto escrito («iTexto! Texto!»), más reclaman los personajes definirse a su manera: « $i$ Te quieres callar ya de una vez y dejarnos ensayar a nuestro aire... ?» (115). Los gritos inútiles de Lev demuestran la imposibilidad de imponer un orden que procede del exterior y también que el texto escrito queda efectivamente desautorizado como medio de concretar las identidades de los personajes. Los gritos de los personajes subrayan que la improvisación artística es el único medio de afirmar sus identidades, de 
definirse en el universo teatral vallejiano. Lo que transcurre en el Cuadro IV es indicativo de un nuevo orden mundial en que la actuación teatral se celebra como artífice y proceso de la concretización del ser.

No cabe la menor duda de que la representación teatral forma la base de la vida en Jindama. La autoridad del arte no es efímera ni vulnerable, sino definitiva e inviolable como se sugiere a continuación en el Cuadro V: «El decorado se ha cerrado sobre ellos» (121). En un mundo cuyas coordenadas reglamentarias son las de una mise-en-abyme, los distintos niveles de existencia se sustituyen sin aviso. Originalmente el director de Jindama, Lev pasa a ser el director de la fantasía de Juanaco. Les pide a los actores que pasen directamente a la escena de la decapitación de Cicerón. Juanaco, a quien la idea de encarnar a Cicerón en el primer cuadro le horrorizó, se mete en el papel sin vacilar. Se dirige a Troncho (Marco Antonio), y reclama: “iAcércate veterano, muestra que sabes herir!» (123). El mundo real del actor ha desaparecido por completo. Según Troncho, Juanaco «tiene que morir. Porque es Cicerón y como asesor fiscal es un delincuente recorrido» (124). Atribuirle a Cicerón la profesión de asesor fiscal de Juanaco quiere decir que los personajes de Cicerón y Juanaco se han fundido y que lo que transcurre en el mundo fantaseado de Juanaco conlleva consecuencias en el mundo de la Jindama dirigida por Lev. Esto confirma lo que asevera Ulrich Broich sobre los personajes posmodernos, que "the characters in postmodernist texts as a rule do not live in a world which mimetically imitates our own world but in textual worlds which imitate other texts» (253). Al superponerse el mundo de la pieza interior y el mundo fantaseado clásico, el mundo de los actores se encoge.

El arte sigue desplegando su poder sobre los personajes en el sexto cuadro: «Se ve en la penumbra cómo el decorado se va transformando, como si fuera una fiera viva» (129). Los personajes son conscientes del poder a que están sometidos. "Como no tengamos cuidado", advierte Tora, "el decorado nos devora» (132). Cuando Juanaco sugiere que «me parece que hace falta salir de aquí», Tora le recuerda que va a ser imposible. "Lo malo», dice, "es que la puerta esa impone». La puerta reacciona como si entendiera: «La puerta realiza extrańos ruidos. Va cambiando escena, se va haciendo la oscuridad e iluminándose el exterior». Cogidos de miedo, «asoman la cabeza por el dintel pero no se atreven a salir» (133). Primero Troncho y luego Reme y Lev ponen sus manos en el dintel con la intención de salir del escenario, pero el escenario se lo prohibe: "Movimiento tembloroso del decorado, agresivo» (134).

Son prisioneros en y de un mundo donde lo real ha sido efectivamente borrado como observan Reme y Juanaco, respectivamente: “iQue nos hemos 
quedado sin sombra!», «QQue nos han robado la sombra!» (135). La falta de sombra es la prueba empírica más certera de la disolución del ser en Jindama. Es la prueba concreta de la aniquilación del «yo», resultado de desempeñar simultáneamente papeles que se contradicen y se contraponen hasta que se invalidan. Citando a Ihab Hassan, podemos concluir que los personajes de Jindama "vacate the traditional self", se pierden en una "reality that is plurally made» y terminan por representar "absence» (19). La caracterización de los personajes en Jindama desafía la noción de una subyacente verdad permanente. El «yo» en Jindama, igual que la realidad, es una entidad frágil, insegura y transitoria.

Hacia finales del drama, los personajes buscan una salida, desesperadamente: "Juanaco sale corriendo hacia la puerta. Pero cuando la va a cruzar, se detiene bruscamente, se tambalea hacia delante, ... extiende las manos para agarrase a las jambas, las toca, grita. Troncho ... corre hacia la puerta, pero cuando la va a cruzar, se desvía». Pero la puerta se impone. Igualmente desesperada e insistente, Reme «se lanza a toda carrera contra la puerta. Pero al intentar cruzarla se produce una descarga» y "permanece inmóvil ». De repente, «Reme empieza a descomponer el gesto, a retorcer la figura. ... Su cuerpo empieza a descomponerse ... Se le doblan muñecas y pies» (136). Las descomposición corpórea de Reme es la evidencia visual del ser fraccionado, del «yo» fragmentado. Lev le implora que vuelva en sí ya que «el ensayo ha terminado» (137). Los síntomas de Reme son los de un personaje que carece de un fondo estabilizador: «Me encuentro totalmente hecha un maremagnum», dice Reme, "de patologías. Si quiero mover un pie, saco la lengua. Si quiero hablar, silencio y si quiero callarme, hablo. ... [C] uando estoy alegre, lloro. Cuando estoy triste, me río» (137-38). La situación parece irreversible: «QQue me sigo deformando» (140), «iQue me desconyuntoooo!» (143). El personaje de Reme, como los de Juanaco, Troncho y Tora, es el sitio de la inestabilidad posmoderna y su desintegración física es la representación teatral de dicha inestabilidad.

Al final del drama, los personajes hacen un esfuerzo colectivo por librarse de su encierro escénico y de Lev, quien fue el que manipuló todo lo que transcurrió en escena. Tora, "sale corriendo para atravesar la puerta .. pero ... por un sistema de poleas unido a un cable ... termina colgada en el aire, gritando». Troncho también "sale corriendo" pero "se va hundiendo como si se tratara de arenas movedizas». Juanaco «se acerca al umbral de la puerta» para ayudar a Troncho pero «se empieza a elevar lentamente hacia el techo, también izado por unos cables» (146). El escenario se prepara para devorarlos. De repente, Reme se repone. Saca un revólver y confronta a Lev. "Dime», insiste, "quién está detrás de todo esto» (147). Lev insiste que 
es un sistema anónimo. Reme vuelve a preguntar: "¿Quién está detrás de todo esto?» Insatisfecha, "le clava la mano sobre la mano con el estilete» y lo dispara (148). La puerta se comienza a cerrar, "la escena empieza a encogerse, paulatinamente, como un animal depredador. ... La puerta se sigue cerrando» (149). Los cuatro se agarran a la puerta y, gracias a su esfuerzo colectivo, "la puerta es vencida por el esfuerzo de los actores». "La abren de par en par» mientras «el decorado vuelve lentamente a su forma natural». Los cuatro actores se ubican por primera vez en la realidad que se encuentra más allá del escenario, su esfuerzo resumido en las palabras que pronuncia Reme y con las cuales se pone fin a la pieza: «Vivir en libertad» (149).

En Jindama, el arte y el personaje teatral constituyen el sitio de la interrogación ontológica de Vallejo. Lo teatral es el texto, contexto, pretexto y subtexto de lo que dramatiza Vallejo en su pieza. Jindama no se apoya en ni es guiado por una realidad autoritaria y orientadora de acuerdo con la autoría teatral tradicional. Los lectores/espectadores de Jindama, como los mismos personajes del drama, se ven confrontados con tres planos artísticos entrelazados, un universo que se expande y se encoge infinitamente al mismo tiempo. Vallejo nos invita a desenredar el tejido narrativo que crea y a utilizar una nueva manera de leer o ver el teatro. Formalmente hablando, se trata de una mise-en-abyme que es, según Moshe Ron, una técnica formal con fines decididamente rebeldes, que «ironically subverts the representational intent» del texto (434) para contender "the hierarchies and ground rules which organize the 'normal' (or classic readable) narrative» (435). En Jindama, el arte engendra el arte y la ilusión se deriva de la ilusión. Ensayar teatro es ensayar a vivir, desempeñar papeles es vivir dichos papeles. El resultado es una multiplicidad de planos de vida en que el sujeto teatral termina descentrado y ofuscado.

La dramaturgia vallejiana se caracteriza por un refinado sentido de composición y complejidad estética e intelectual. Para Alfonso Vallejo, el teatro es un procedimiento dinámico de fuerzas artísticas y cerebrales cuyos atributos definitorios son la relación problemática entre la presentación y la representación, la (in)compatibilidad de la estructura y la historia, la ambigüedad de lo espacial y lo temporal, la contigüidad y la confluencia de lo real y lo imaginario y la construcción antirrealista del personaje, todas características relacionadas con el ímpetu esencialmente existencial y ontológico del teatro de los siglos XX y XXI. Es una escritura teatral que se subscribe a una visión marcadamente posmoderna de la realidad y del arte y que, como el Don Quijote de Cervantes, muestra el peligro de nutrirse y sostenerse de ilusiones. 


\section{Obras citadas}

Baudrillard, Jean, Selected Writings. Ed. Mark Poster. Stanford, Stanford UP, 1988.

Bertens, Hans, "Postmodern Characterization and the Intrusion of Language». Ed. Douwe Fokkema and Matei Calinescu. Exploring Postmodernism. Amsterdam, John Benjamins, 1987, 139-59.

Broich, Ulrich, "Intertextuality». International Postmodernism: Theory and Literary Practice. Ed. Hans Bertens and Douwe Fokkema. Amsterdam, John Benjamins, 1997, 249-55.

Cardwell, Richard A., «Beyond the Mirror and the Lamp: Symbolist Frames and Spaces». Romance Quarterly 36.3, 1989, 271-80.

Coco, Emilio, Teatro spagnolo contemporaneo. Vol. II. Alessandria, Edizione dell'Orso, 2000.

Corrigan, Robert W., "The Search for New Endings, The Theatre in Search of a Fix, Part III", Theatre Journal, 36.2, 1984, 153-63.

Docherty, Thomas, Reading (Absent) Character. Towards a Theory of Characterization in Fiction. Oxford, Clarendon Press, 1983.

Gutiérrez Carbajo, Francisco, Teatro contemporáneo: Alfonso Vallejo. Madrid, Universidad Nacional de Educación a Distancia, 2001.

Hassan, Ihab, «Pluralism in Postmodern Perspective». Exploring Postmodernism. Ed. Matei Calinescu and Douwe Fokkema. Amsterdam, John Benjamins, 1987, 17-39.

Hutcheon, Linda, A Poetics of Postmodernism: History, Theory, Fiction. London, Routledge, 1988.

Kaye, Nick, Postmodernism and Performance. New York, St Martin's Press, 1994.

Lethen, Helmut, "Modernism Cut in Half: The Exclusion of the Avant-Garde and the Debate on Postmodernism». Approaching Postmodernism. Ed. Douwe Fokkema and Hans Bertens. Amsterdam, John Benjamins, 1986. 233-38.

Malkin, Jeanette R., Memory-Theater and Postmodern Drama. Ann Arbor, The U of Michigan P, 1999.

Malpas, Simon, The Postmodern. London, Routledge, 2005.

Martín, Sabas, «El teatro de Alfonso Vallejo». Cuadernos hispanoamericanos 416, 1985, 151-57.

McHale, Brian, Constructing Postmodernism. London, Routledge, 1992.

Murphy, Richard, Theorizing the Avant-Garde. Modernism, Expressionism, and the Problem of Postmodernity. Cambridge, Cambridge UP, 1999.

Rao, N. M. «The Self-Commenting Drama of Our Times». The Aligarth Journal of English Studies 9.2, 1984, 215-26.

Ron, Moshe, "The Restricted Abyss. Nine Problems in the Theory of Mise-enabyme». Poetics Today 8.2, 1987, 417-38. 
Schlueter, June, Metafictional Characters in Modern Drama. New York, Columbia UP, 1979.

Schulte-Sasse, Jochen. «Modernity and Modernism, Postmodernity and Postmodernism: Framing the Issue». Cultural Critique 5, 1987, 5-22.

Sim, Stuart, ed. The Routledge Companion to Postmodernism. London, Routledge, 2001.

Vallejo, Alfonso, Jindama. Salobreña, Granada, Alhulia, 1998.

Waugh, Patricia, Metafiction, The Theory and Practice of Self-Conscious Literature. London, Methuen, 1984. 
